SMALL INTESTINE

\title{
Homeostatic regulation of zinc transporters in the human small intestine by dietary zinc supplementation
}

\author{
R A Cragg, S R Phillips, J M Piper, J S Varma, F C Campbell, J C Mathers, D Ford
}

Gut 2005;54:469-478. doi: 10.1136/gut.2004.041962

See end of article for authors' affiliations

Correspondence to: Dr D Ford, Institute for Cell and Molecular Biosciences, University of Newcastle, The Medical School, Newcastle upon Tyne NE2 4HH, UK; dianne.ford@ ncl.ac.uk

Revised version received 4 October 2004

Accepted for publication 12 October 2004
Background: The role of intestinal transporter regulation in optimising nutrient absorption has been studied extensively in rodent and cell line models but not in human subjects.

Aims: The aim of the present study was to investigate the response in vivo of zinc transporters in the human enterocyte to dietary zinc supplementation.

Subjects: Eighteen patients who had previously undergone ileostomy, all free of any symptoms of inflammatory bowel disease.

Methods: Subjects took a daily zinc supplement of $25 \mathrm{mg}$ for 14 days in a double blind, placebo controlled, crossover trial. The effect of the supplement on expression in ileal biopsies of the zinc transporters SLC30A1, SLC30A4, SLC30A5, SLC39A1, SLC39A4, and metallothionein was measured by reverse transcription-polymerase chain reaction RT-PCR. Expression of SLC30A1, SLC30A5, and SLC39A4 was also examined by immunoblotting.

Results: The zinc supplement reduced SLC30A1 mRNA (1.4-fold) together with SLC30A1, SLC30A5, and SLC39A4 protein (1.8-fold, 3.7-fold, and to undetectable levels, respectively) in ileal mucosa and increased metallothionein mRNA (1.7-fold). The supplement had no effect on expression of SLC30A4 or SLC39A1 mRNA. Localisation of SLC30A5 at the apical human enterocyte/colonocyte membrane and also at the apical membrane of Caco-2 cells was demonstrated by immunohistochemistry. Commensurate with these observations in zinc supplemented human subjects, SLC30A1, SLC30A5, and SLC39A4 mRNA and protein were reduced in Caco- 2 cells cultured at $200 \mu \mathrm{M}$ compared with $100 \mu \mathrm{M}$ zinc.

Conclusions: These observations indicate that, in response to variations in dietary zinc intakes, regulated expression of plasma membrane zinc transporters in the human intestine contributes to maintenance of zinc status.
$\mathrm{E}$ xpression of nutrient transporters in the enterocyte in rodent and cell line models responds to nutrient load. Observed patterns of response for essential nutrients and/or nutrients that are toxic in excess, such as zinc and iron, are generally consistent with maintenance of body nutrient status under conditions of variable intake. ${ }^{12}$ For example, expression of the divalent metal ion transporter DMTl, involved in iron absorption, is increased in the intestine of rats fed an iron deficient diet. ${ }^{3}$ For transporters of non-toxic nutrients whose principal role is to provide energy, such as glucose, upregulation of expression by increased availability of substrate nutrient avoids the energy costs of transporter maintenance in the absence of substrate supply. ${ }^{2}$

Zinc is an essential micronutrient which, as a catalytic and/ or structural cofactor for several hundred metalloproteins, is involved in a diverse array of cellular functions. Regulated intestinal absorption of dietary zinc is believed to be important in zinc homeostasis. Of the zinc transporters cloned to date and expressed in the intestine, localisation and transport function of SLC30Al, SLC30A5, and SLC39A4 are consistent with a role in dietary zinc absorption. Apical localisation of a c-myc tagged construct corresponding to one of two identified splice variants of SLC30A5 $5^{45}$ when expressed in Caco-2 cells, ${ }^{4}$ coupled with a demonstrated ability of the same splice variant to mediate cellular zinc uptake, ${ }^{4}$ indicates the involvement of this transporter in the transport of zinc from the intestinal lumen into the enterocyte. The discovery of SLC39A4 as the gene mutated in most cases of acrodermatitis enteropathica, characterised by impaired zinc absorption, identified this transporter as having a role in the uptake of zinc from the intestinal lumen. ${ }^{67}$ The corresponding mouse gene product is localised at the apical enterocyte membrane ${ }^{7}$ and when expressed in human embryonic kidney (HEK293) cells has been shown to mediate zinc uptake. ${ }^{8}$ A role for SLC30Al in the efflux of zinc from the enterocyte into the serosa is supported by its basolateral location in rat intestine, as established using an antipeptide antibody, ${ }^{9}$ coupled with functional evidence indicating a role in cellular zinc efflux. ${ }^{10}$

Upregulation of Slc30al mRNA and protein in the rat small intestine was observed on a zinc supplemented compared with a zinc adequate diet but a zinc deficient diet had no effect on Slc30al expression in this model. ${ }^{911}$ In contrast, Slc39a4 mRNA was increased in the small intestine of mice fed a zinc deficient compared with a zinc adequate diet and the effect was reversed on switching diets from zinc deficient to zinc supplemented. ${ }^{8}$ In the human intestinal Caco- 2 cell line, we demonstrated that increasing the extracellular zinc concentration from $3 \mu \mathrm{M}$ to $100 \mu \mathrm{M}$ for seven days resulted in increased levels of mRNAs corresponding to the zinc transporters SLC30Al, SLC30A4, ${ }^{12}$ and SLC30A5. ${ }^{4}$ Regulation by dietary zinc of other zinc transporters expressed in the intestine has also been studied in rodent models. Levels in the small intestine of mRNA species corresponding to the zinc transporter Slc30a2, believed to function in the sequestration of zinc by intracellular vesicles, ${ }^{13}$ were increased by moderate zinc supplementation and reduced on severe zinc depletion. ${ }^{11}$ However, levels in rat small

Abbreviations: $B B M V$, brush border membrane vesicles; $B M R$, basal metabolic rate; MT, metallothionein; RT-PCR, reverse transcriptionpolymerase chain reaction; SDS-PAGE, sodium dodecyl sulphatepolyacrylamide gel electrophoresis; PBS, phosphate buffered saline 
intestine of the mRNA coding for Slc30a4, which is also believed to play a role in zinc transport into intracellular vesicles, ${ }^{14}$ were not changed by moderate zinc supplementation or severe dietary zinc depletion. ${ }^{11}$ Dietary zinc deficiency in pregnant mice had no effect on expression in the intestine of the zinc transporters Slc39al and Slc39a3, which act to mediate zinc uptake when expressed in transfected HEK293 cells but whose pattern of expression and function in the intestine are unknown. ${ }^{15}$

Studies in the rat using both isolated intestinal brush border membrane vesicles (BBMV) ${ }^{16}$ and isolated vascularly perfused intestine ${ }^{17}{ }^{18}$ have demonstrated increased maximal rates of zinc absorption after dietary zinc depletion. Increased expression at the brush border membrane of zinc transporters involved in zinc uptake at lower levels of dietary zinc intake, such as reported for Slc39a4 in the mouse, ${ }^{8}$ would offer a molecular explanation for these functional observations.

Studies in human volunteers involving supplementation or modification of the diet are required to establish the relevance in the human intestine in vivo of regulatory responses of nutrient transporter genes to their substrates in general, and of zinc transporter genes to dietary zinc intake in particular. Practical and ethical difficulties associated with sampling of the human intestinal mucosa are probably responsible for the current paucity of direct evidence in vivo of regulatory responses of nutrient transporter genes to dietary nutrient intake in humans. We report here for the first time data on transporter expression in intestinal mucosa quantified at the molecular level in human volunteers in response to nutrient supplementation.

\section{MATERIALS AND METHODS Study design}

The study was a double blind, randomised, placebo controlled, crossover trial in which the active treatment was a daily supplement of $25 \mathrm{mg} \mathrm{Zn}^{2+}$ (as a single capsule of zinc sulphate) or placebo (sodium chloride), given for 14 days with a washout period of at least 28 days between trials. Assignment to supplement or placebo was according to a computer generated randomisation scheme performed by the study pharmacist who retained codes until all analysis was complete. None of the investigators or patients had access to the randomisation codes. Power calculation indicated that a sample size of 16 subjects would provide $80 \%$ power for detection of a $23 \%$ change in level of zinc transporter expression assuming a standard deviation of differences of 0.3 based on a normalised population mean of 1 .

The study was approved by the Joint Ethics Committee of the Newcastle and North Tyneside Health Authority, University of Newcastle and University of Northumbria at Newcastle, UK. Written informed consent was obtained from all participants.

\section{Participants}

Participants ( 12 females, six males) were aged 33-76 years and had previously undergone ileostomy with minimal ileal resection. All subjects were free of any symptoms of previous inflammatory bowel disease and none was undergoing any clinical procedure for treatment of their previous disease. Participants attended a clinic at the Royal Victoria Infirmary, Newcastle upon Tyne, UK, between March 2000 and November 2000 before and after each trial period solely for the purpose of participating in the study.

Supplement or placebo was dispensed by the investigator at the clinic and subjects were instructed to take one capsule daily with food. Samples of $5 \mathrm{ml}$ venous blood were collected in each of EDTA, heparinised, and untreated containers on each visit and pinch biopsies of ileal mucosa (3$4 \mathrm{~mm} \times 1 \mathrm{~mm}^{2}$ ) were taken through the stoma after each trial period and immediately frozen in liquid nitrogen.

\section{Analysis of nutrient intake}

Participants recorded all food and beverage intake over seven days of each 14 day trial period. Portion sizes were quantified by a food photographic atlas ${ }^{19}$ during the post trial visit to the clinic. Food $\operatorname{codes}^{20}$ were assigned and nutrient intakes were calculated using inhouse custom software and standard UK food composition tables.

Measurement of plasma and serum zinc concentration For preparation of plasma, $5 \mathrm{ml}$ of blood collected into a heparinised tube were centrifuged at $2500 \mathrm{~g}$ for 15 minutes at $4^{\circ} \mathrm{C}$. The resultant clear plasma layer was removed and stored at $-80^{\circ} \mathrm{C}$. For preparation of serum, an untreated glass tube containing $5 \mathrm{ml}$ of blood was placed on its side for 30 minutes at room temperature to allow clotting to occur. The sample was then centrifuged at $2500 \mathrm{rpm}$ for 15 minutes at $4^{\circ} \mathrm{C}$ and the top layer subsequently removed and stored at $-80^{\circ} \mathrm{C}$. Plasma and serum were diluted $1: 10$ in deionised water and zinc concentration was measured using a Unicam 701 inductively coupled plasma optical emission spectrometer.

Table 1 Primers and conditions for polymerase chain reaction (PCR)

\begin{tabular}{|c|c|c|c|}
\hline Transcript & Forward and reverse primers & $\begin{array}{l}\text { Genbank } \\
\text { accession No }\end{array}$ & No of cycles \\
\hline SLC30A1 & $\begin{array}{l}{ }^{1251} \text { TCAGCCTGAATTTGCTAG }{ }^{1268} \\
{ }^{1514} \text { GATCAGGTTGTITGITGGC }{ }^{1494}\end{array}$ & NM_021194 & 30 \\
\hline SLC30A4 & $\begin{array}{l}{ }^{763} \text { CTGACCTAAGCGCCATCATACTCACCC }^{789} \\
{ }^{1141} \text { AATGCAGCTCTCACTGCC }{ }^{1124}\end{array}$ & NM_013309 & 30 \\
\hline SLC30A5 & $\begin{array}{l}{ }^{1945} \text { GGTGGAGGCATGAATGCTA } \\
2166 \text { TCTGGTGGCAATCTCAGG } \\
2148\end{array}$ & NM_022902 & 30 \\
\hline SLC39A1 & $\begin{array}{l}{ }^{447} \text { TGAGAGTCACTGGAGCTACCA }^{467} \\
{ }^{732} \text { AGGAGACAAGTGGCCAAAAA }^{713}\end{array}$ & NM_014437 & 30 \\
\hline SLC39A4 & $\begin{array}{l}{ }^{328} \text { GCCTGCGGGGTCAGGGCTGC }^{347} \\
{ }^{518} \text { GGCCCGGGGTCAGGGCCTTG }^{499}\end{array}$ & NM_130849 & 30 \\
\hline MT & $\begin{array}{l}{ }^{71} \text { ATGGACCCCAACTGCTCCC } \\
{ }^{256} \text { TCAGGCACAGCAGCTGCAC }^{238}\end{array}$ & NM_005946 & *25, †30 \\
\hline$\beta$-actin & $\begin{array}{l}{ }^{894} \text { TCCACGAAACTACCTTCAAC }^{913} \\
{ }^{1498} \text { TाTAGGATGGCAAGGGAC }\end{array}$ & NM_001101 & $\ddagger 25, \S 30$ \\
\hline
\end{tabular}

All primer sequences are given as $5^{\prime}-3^{\prime}$. Superscript numerals indicate the primer annealing positions with respect to the cDNA sequence deposited under the stated Genbank accession number.

Thermal cycling parameters for all PCR reactions were: $95^{\circ} \mathrm{C}$ five minutes followed by $194^{\circ} \mathrm{C} 30$ seconds, $55^{\circ} \mathrm{C}$ 30 seconds, $72^{\circ} \mathrm{C} 90$ seconds) for the stated number of cycles.

Symbols indicate where the cycle number was altered depending on the RNA source: *poly- $A^{+}$RNA from biopsies; tpoly-A $\mathrm{A}^{+}$RNA from monocytes; łpoly-A $\mathrm{A}^{+}$RNA from biopsies and monocytes; and Stotal RNA from Caco- 2 cells. 


\section{Culture of Caco- 2 cells}

Caco-2 cells (passage 30+) were cultured as described previously. ${ }^{4}$ For growth at increased concentrations of zinc, $100 \mu \mathrm{M}$ or $200 \mu \mathrm{M} \mathrm{ZnCl}{ }_{2}$ was added to the culture medium of confluent cells ( 14 days post seeding) and cells were maintained in this medium for a further three days before harvesting RNA.

\section{Preparation of RNA}

Monocytes were prepared using Nycoprep 1.068 (Sigma, St Louis, Missouri, USA) following the manufacturer's instructions. Total RNA was prepared from Caco-2 cells, blood monocytes, and human intestinal biopsies using RNAzolB (Biogenesis, Poole, Dorset, UK), following the manufacturer's instructions. Poly- $\mathrm{A}^{+}$RNA was isolated from total RNA using the NucleoTrap mRNA kit (Macherey-Nagel, Düren, Germany), following the manufacturer's instructions.

\section{Reverse transcription-polymerase chain reaction (RT-PCR)}

Total RNA from Caco-2 cells was treated with DNAse I (Invitrogen, Paisley, Scotland, UK) according to the manufacturer's instructions. DNAse I was inactivated by addition of EDTA to $2.5 \mathrm{mM}$, followed by a 10 minute incubation at $65^{\circ} \mathrm{C}$. Reverse transcription of poly- $\mathrm{A}^{+} \mathrm{RNA}(1 \mu \mathrm{g})$ or DNAse treated RNA $(1 \mu \mathrm{g})$ was carried out using Superscript III reverse transcriptase (Invitrogen). Samples were then amplified by PCR using Thermo-Start DNA polymerases (Abgene Ltd, Epsom, Surrey, UK). Specific primers and cycling parameters are given below in table 1 . PCR products were resolved on $2 \%$ agarose gels containing $10 \mu \mathrm{g} / \mathrm{ml}$ ethidium bromide and visualised using a BTS-26M imager (Uvitec Ltd, Cambridge, UK). Relative band intensity was quantified using UviPhotoMW image analysis software.

\section{Preparation of brush border membrane vesicles (BBMV) from human jejunum}

Human jejunum was obtained from a cadaver donor with approval from the Committee on Medical Ethics, Tayside Health Board. BBMV were prepared from frozen human jejunum tissue using a combination of cation precipitation and differential centrifugation, as described previously. ${ }^{21} 22$ Activity of alkaline phosphatase was assayed at pH 9.0 using p-nitrophenyl phosphate (Sigma) as substrate. ${ }^{23}$ Enzyme activity was expressed as $\mu \mathrm{mol} / \mathrm{min} / \mathrm{mg}$ protein. Prepared BBMV were stored at $-80^{\circ} \mathrm{C}$.

\section{Protein assay}

Protein was determined by its capacity to bind Coomassie blue (Bradford assay) using a protein assay kit (Bio-rad Laboratories, Hercules, California, USA) with bovine serum albumin (Sigma) as standard.

\section{Generation of antibodies}

Rabbit anti-SLC30Al was raised against a synthetic peptide corresponding to amino acids 495-508 of the human gene product (EIKNMPNKQPESSL). Rabbit anti-SLC30A5 was raised against a synthetic peptide corresponding to amino acids 190-211 of the human splice variant 1 gene product and to amino acids $361-382$ of the human splice variant 2 gene product (ILSSPSKRGQKGTLIGYSPEGT). Both peptides included a $\mathrm{C}$ terminal cysteine and were conjugated to a SulfoLink column (Pierce Biotechnology Inc, Rockford, Illinois, USA) for affinity purification of antibody, following the manufacturer's instructions. Affinity purified rabbit antiSLC39A4 anti-peptide antibody was a gift from Dr S Kury, LEPA, Nantes, France.

\section{Heterologous expression of SLC30A1, SLC30A5, and SLC39A4 protein}

The region of SLC30Al between amino acid resides 334 and 507 was amplified by RT-PCR from Caco-2 mRNA using the primers TCA GAT TAC CGA GGG ATG ${ }^{1000}$ GCA TTA CTT AAG GAA TCT $G^{1018}$ and GAG CTC GGG CCC ${ }^{1521}$ CAA AGA TGA TTC AGG TTG ${ }^{1504}$ (numbering according to the sequence under accession number NM_021194; restriction endonuclease cleavage sites for $K p n \mathrm{I}^{-}$and ApaI included six bases from the $5^{\prime}$ end of the forward and reverse primers, respectively, and an ATG start codon and Kozak consensus sequence incorporated into the forward primer). PCR was over 30 cycles using the thermal cycling parameters: $94^{\circ} \mathrm{C}$ for 30 seconds, $55^{\circ} \mathrm{C}$ for 30 seconds, and $72^{\circ} \mathrm{C}$ for 90 seconds. The product was subcloned into the vector pCR2.1-TOPO (Invitrogen). The SLC30Al sequence was excised using ApaI and KpnI and subcloned into vector pEGFP-N2 (BD Biosciences Clontech, Palo Alto, California, USA) to give the plasmid pSLC30AlGFP. The full ORF of SLC39A4 was amplified from the vector pGEMZIP4 (a gift from Dr S Kury, LEPA, Nantes, France) using the primers CAT AGA TCT $^{95}$ CTG AGT ATG GCG TCC ${ }^{109}$ and CAT AAG CTT ${ }^{2032}$ GTC ATC CTC GTA CAG GG $^{2016}$ (numbering according to the sequence under accession number NM_130849; restriction endonuclease cleavage sites for BgIII and HindIII included three bases from the $5^{\prime}$ end of the forward and reverse primers, respectively). PCR was over 30 cycles using the thermal cycling parameters: $94^{\circ} \mathrm{C}$ for 30 seconds, $60^{\circ} \mathrm{C}$ for 30 seconds, and $72^{\circ} \mathrm{C}$ for 120 seconds. The product was cleaved with BglII and HindIII and subcloned into the vector pEGFP-Nl (Clontech) to give the plasmid pSLC39A4GFP. Endotoxin free plasmid preparations (Qiagen Endo-Free Plasmid Maxi Kit; Qiagen Ltd, Crawley, Surrey, UK) of pSLC30AlGFP and pSLC39A4GFP were used to transfect COS-7 cells using a calcium phosphate/DNA precipitation protocol, as previously described. ${ }^{4}$ Cells were harvested for immunoblotting 48 hours after transfection. Xenopus laevis oocytes were injected with in vitro transcribed SLC30A5 RNA (cRNA) or with water, as previously described. ${ }^{4}$

\section{Immunoblotting}

Caco-2 or COS-7 cell pellets harvested from flasks using a cell scraper, BBMV, Xenopus laevis oocytes, or human mucosal biopsies were resuspended by vortexing or homogenised by hand in a buffer containing $100 \mathrm{mM} \mathrm{NaCl}, 10 \mathrm{mM}$ Tris $\mathrm{HCl}$ ( $\mathrm{pH} 7.6), 1 \mathrm{mM}$ EDTA, $1 \mu \mathrm{g} / \mathrm{ml}$ aprotinin, and $100 \mu \mathrm{g} / \mathrm{ml}$ phenylmethyl sulphonyl fluoride, and stored at $-80^{\circ} \mathrm{C}$. Samples were diluted in an equal volume of a buffer containing $100 \mathrm{mM}$ Tris $\mathrm{HCl}$ (pH 6.8), $200 \mathrm{mM} \beta$-mercaptoethanol, $4 \%$ sodium dodecyl sulphate, and $20 \%$ glycerol. The suspension was boiled for 10 minutes and then centrifuged at $13000 \mathrm{~g}$ for 10 minutes. The supernatant was assayed for its protein concentration. Total protein (7$50 \mu \mathrm{g}$, as indicated in the results section) was subjected to sodium dodecyl sulphate-polyacrylamide gel electrophoresis (SDS-PAGE) on a 7.5\% acrylamide gel. Proteins were transferred onto PVDF membranes (Hybond P; Amersham Biosciences UK Ltd, Little Chalfont, Buckinghamshire, UK) and incubated with affinity purified anti-SLC30Al (1:100), anti-SLC30A5 (1:100), or anti-SLC39A4 antibodies (1:100 or $1: 200)$, or with anti-SLC39A4 rabbit serum (1:500), in $1 \times$ phosphate buffered saline (PBS), pH 7.4, containing 0.01\% Tween 20 and $5 \%$ non-fat milk powder overnight at $4{ }^{\circ} \mathrm{C}$ followed by incubation for one hour at room temperature with a horseradish peroxidase conjugated goat antirabbit IgG secondary antibody (1:5000 dilution) (Sigma). Antibody binding was visualised as peroxidase activity using ECLPlus (Amersham Pharmacia Biotech) according to the manufacturer's instructions. Densitometric analysis of band 
A

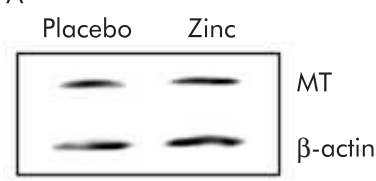

B

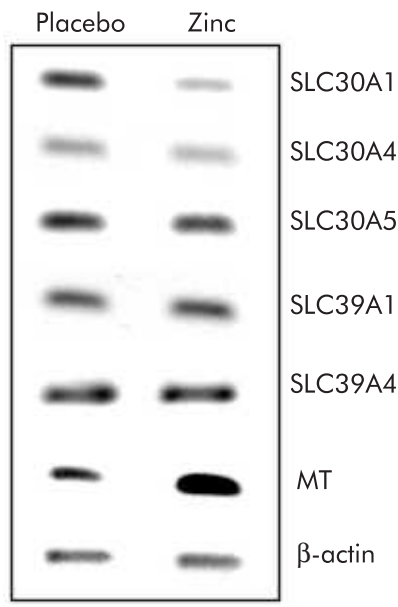

C

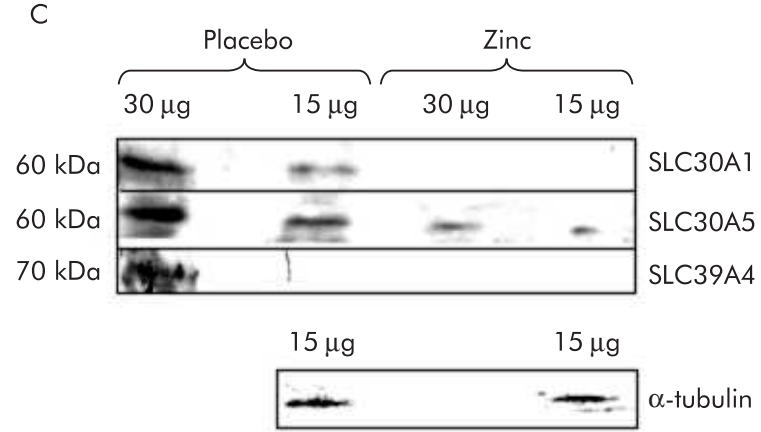

Figure 1 Comparison of the expression of zinc related genes in peripheral blood monocytes and biopsies of human small intestine taken either after zinc supplementation or administration of placebo. (A) Reverse transcription-polymerase chain reaction (RT-PCR) products generated using primers specific to the transcripts indicated from poly- $A^{+}$RNA prepared from total monocyte RNA pooled from 17 volunteers. (B) RT-PCR products generated using primers specific to the transcripts indicated from poly- $A^{+}$RNA prepared from total RNA pooled from single intestinal biopsies from 17 volunteers. For $(A)$ and $(B)$, products generated from poly- $A^{+}$RNA prepared from biopsies following administration of placebo or zinc supplement are shown as indicated. PCR was carried out over a non-saturating number of cycles and thus band intensity is representative of the quantity of the specific transcript in the RNA sample. Three independent analyses of the relative expression level of each transcript in the two samples gave comparable results. MT, metallothionein. (C) Analysis by immunoblotting using antipeptide antibodies of expression in pooled biopsies from each of 17 volunteers of the zinc transporters SLC30A1, SLC30A5, and SLC39A4, and of $\alpha$-tubulin, as indicated. For each sample either $15 \mu \mathrm{g}$ or $30 \mu \mathrm{g}$ of protein, as determined by Bradford analysis, were resolved by sodium dodecyl sulphate-polyacrylamide gel electrophoresis prior to blotting. The result of analysis of samples prepared from biopsies following administration of placebo or the zinc supplement are shown as indicated.

intensities was performed using UviPhotoMW image analysis software.

\section{Immunohistochemistry and immunocytochemistry}

Formalin fixed human jejunum, ileum, and colon sections were purchased from Peterborough Hospital Trust tissue bank. Tissue sections were permeabilised by immersion of the slide for five minutes in 100\% xylene, 100\% ethanol, and $95 \%$ ethanol. The antigens were exposed by heating the slides three times for five minutes in a microwave, in $1 \mathrm{mM}$ sodium citrate buffer ( $\mathrm{pH}$ 6.0). Caco-2 cells grown for 14 days on polycarbonate filters were fixed with $100 \%$ methanol for five minutes at room temperature. Sections or cells were incubated in blocking solution ( $10 \%$ goat serum in PBS) for 30 minutes at room temperature. After incubation overnight at $4^{\circ} \mathrm{C}$ with anti-SLC30A5 antibody $(1: 100)$, slides were washed in PBS and samples were then incubated with FITC conjugated goat antirabbit IgG (1:500) (Sigma) for one hour at room temperature. Caco-2 cells were subsequently treated with propidium iodide $(5 \mu \mathrm{g} / \mathrm{ml})$ to reveal nuclear staining or with a chromogenic alkaline phosphatase stain (Alkaline Phosphatase Substrate Kit 1; Vector Laboratories, Burlingame, California, USA). Sections or filters were mounted under a sealed coverslip and visualised using a Leica confocal microscope.

\section{Statistical analysis}

Where appropriate, data were analysed by repeated measures ANOVA or paired $t$ test using Graphpad software and expressed as mean (SD). Statistical significance was set at $\mathrm{p}<0.05$.

\section{RESULTS}

The regulatory response of zinc transporters in the human intestinal mucosa to changes in dietary zinc intake was studied in volunteers who had previously undergone ileostomy and were free of any symptoms of previous inflammatory bowel disease and who attended a clinic solely for the purpose of participating in the study. Eighteen subjects ( 12 females) were recruited to a double blind, placebo controlled, randomised, crossover study in which the active treatment was a daily supplement of $25 \mathrm{mg} \mathrm{Zn}^{2+}$ (as zinc sulphate) or placebo (sodium chloride), given for 14 days with a washout period of at least 28 days between trials. One subject (female, aged 51 years with a previous diagnosis of ulcerative colitis) failed to complete the study. Volunteers who completed the trial were aged 33-76 years (mean 51 years) with a body mass index of 15.1-43.5 (mean 25.3). Subjects recorded all food and beverage intake over seven days of each trial period. Analysis of these records indicated mean daily zinc intakes of 8.2 (SD 2.4) $\mathrm{mg}$ while taking placebo and 33.0 (SD 2.2) mg (including the zinc supplement) while taking the zinc supplement. Analysis by repeated measures ANOVA indicated that pre- and post intervention serum and plasma zinc concentrations did not differ significantly (plasma: 19.2 (4.9) $\mu \mathrm{M}$ (mean (SD)) compared with $20.4(6.4) \mu \mathrm{M}$ before and after the zinc supplement, respectively; serum: 17.2 (2.5) $\mu \mathrm{M}$ compared 

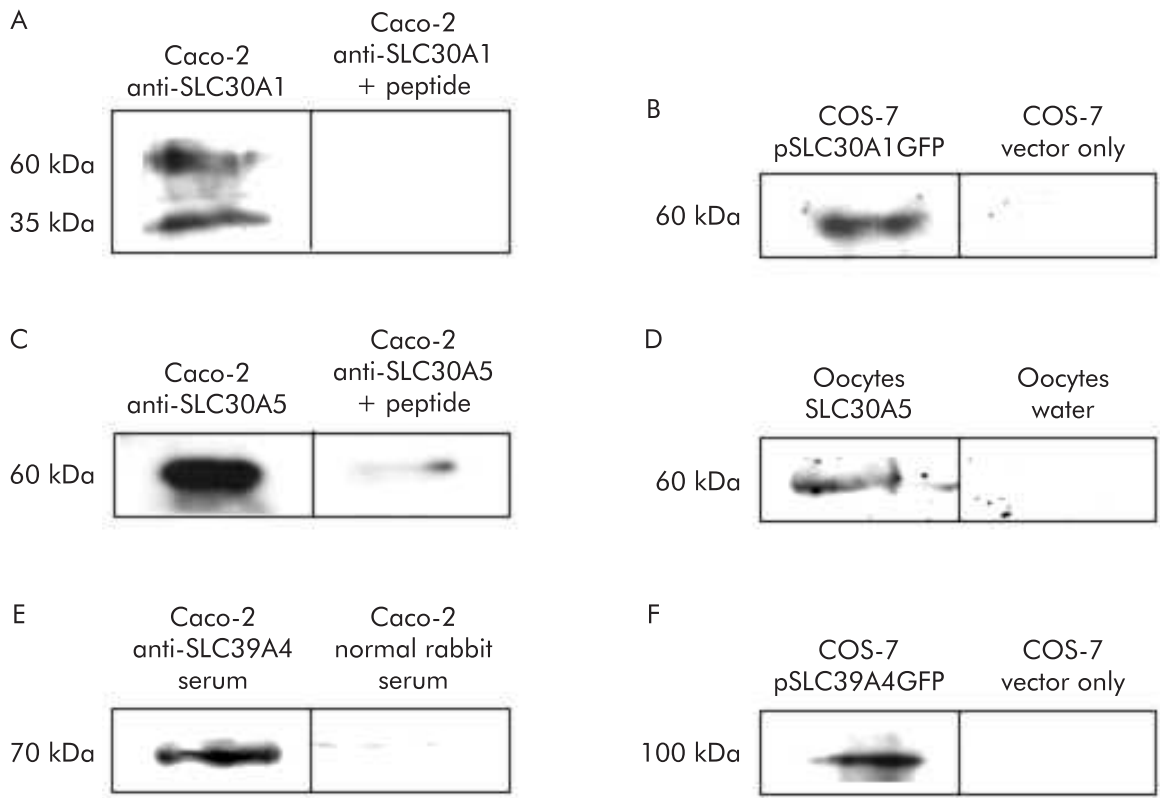

Figure 2 Verification of specificity and target protein reactivity of anti-SLC30A1, anti-SLC30A5, and anti-SLC39A4 antipeptide antibodies. (A, B) Immunoblots using anti-SLC30A1 antibody. (C, D) Immunoblots using anti-SLC30A5 antibody. (E, F) Immunoblots using anti-SLC39A4 antibody. Data for Caco-2 cells $(A, C, E)$ were obtained from untransfected cells. Data for COS-7 cells $(B, D)$ were obtained from cells transfected with the plasmid constructs indicated. (A) Protein $(7 \mu \mathrm{g})$ extracted from Caco-2 cells was run in each lane and the blot was incubated with affinity purified anti-SLC30A1 antibody at a dilution of $1: 100$ or with the same amount of antibody plus $2.5 \mu \mathrm{g} / \mathrm{ml}$ of the immunising peptide, as indicated. (B) Protein was prepared from COS-7 cells transfected with a plasmid construct (pSLC30A1GFP) from which the C terminal region of SLC30Al between amino acid residues 334 and 507, inclusive, was expressed as an N terminal fusion to GFP or with vector only (plasmid pEGFPN), as indicated; $20 \mu \mathrm{g}$ were run in each lane and the blot was incubated with a 1:100 dilution of affinity purified anti-SLC30A1 antibody. (C) Protein (7 $\mu \mathrm{g}$ ) extracted from Caco-2 cells was run in each lane and the blot was incubated with affinity purified anti-SLC30A5 antibody at a dilution of 1:100 or with the same amount of antibody plus $2.5 \mu \mathrm{g} / \mathrm{ml}$ of the immunising peptide, as indicated. (D) Protein was prepared from Xenopus laevis oocytes injected with SLC30A5 cRNA or with water (10 of each), as indicated; $20 \mu \mathrm{g}$ were run in each lane and the blot was incubated with a 1:100 dilution of affinity purified anti-SLC30A5 antibody. (E) Protein $(7 \mu \mathrm{g}$ ) extracted from Caco-2 cells was run in each lane and the blot was incubated with a 1:500 dilution of serum from a rabbit treated with the immunising peptide or with a 1:500 dilution of normal rabbit serum, as indicated. (F) Protein was prepared from COS-7 cells transfected with a plasmid construct (pSLC39A4GFP) from which SLC39A4 was expressed as an N terminal fusion to GFP or with vector only (plasmid pEGFPN), as indicated; $20 \mu \mathrm{g}$ were run in each lane and the blot was incubated with a 1:200 dilution of affinity purified anti-SLC39A4 antibody.

with 20.0 (6.2) $\mu \mathrm{M}$ before and after the zinc supplement, respectively).

Levels of monocyte metallothionein mRNA, cited as a potential marker of zinc status, ${ }^{24}$ before and after both trial periods (zinc and placebo) were compared by analysis of poly- $\mathrm{A}^{+}$RNA prepared from pooled total RNA by RT-PCR. PCR was carried out over a number of cycles limited such that the reaction did not reach the plateau phase to ensure that final product yield was sensitive to the amount of cDNA in the template sample. Amplification of the same cDNA samples using primers specific for $\beta$-actin, also over a non-saturating number of cycles, served as a control to ensure equal addition of cDNA to both reactions. The results (fig lA) indicated no change in monocyte metallothionein mRNA levels under the conditions tested.

Biopsies of ileal mucosa were taken through the stoma at the end of each trial period. Levels of specific mRNA species in poly- $\mathrm{A}^{+}$mRNA prepared from pooled total RNA from one biopsy from each volunteer were compared between the two trial periods by RT-PCR. To ensure that final product yield was sensitive to the amount of cDNA in the template sample, the number of PCR cycles was limited such that that the reaction did not reach the plateau phase. Amplification of the same cDNA samples using primers specific for $\beta$-actin, also over a non-saturating number of cycles, served as a control to ensure equal addition of cDNA to all reactions. Visual inspection of the results indicated no change in levels of SLC30A4, SLC30A5, SLC39A1, or SLC39A4 mRNAs in response to the zinc supplement but reduced levels of SLC30Al mRNA and increased levels of metallothionein mRNA (fig 1B). Repetition of the same analysis twice more yielded the same results. Based on all three analyses, densitometric quantification of relative band intensities showed that the ratio of the signal intensities for zinc compared with placebo was $0.70(0.07)$ (mean (SD)) for SLC30Al and 1.65 (0.31) for metallothionein.

Levels of SLC30Al, SLC30A5, and SLC39A4 protein in samples prepared from pooled biopsies (one biopsy per volunteer) were measured by immunoblotting using affinity purified antibodies raised in rabbits against synthetic peptides corresponding to regions of the relevant proteins. The predominant band to which antibodies bound was approximately $60 \mathrm{kDa}$ for SLC30Al and SLC30A5 and for SLC39A4 approximately $70 \mathrm{kDa}$. Equal quantities of both protein samples, as determined by measurement of protein concentration using the Bradford assay, were resolved by SDS-PAGE prior to blotting. Equal loading was confirmed by staining a duplicate gel with Coomassie blue and complete transfer of protein to the membrane was confirmed by staining the gel after transfer with Coomassie blue. Equal loading and also integrity of both samples was further confirmed by immunoprobing of a replica blot with an anti$\alpha$-tubulin antibody (fig 1C). Visual inspection of immunoblots (fig 1C) revealed higher levels of expression of all zinc transporters following administration of placebo than following the zinc supplement. For SLC30Al, this observation was commensurate with the measured change in mRNA levels. Densitometric quantification of relative band intensities indicated an approximate 3.7-fold reduction in SLC30Al expression at the higher zinc intake (mean 0.27 (SD 0.02) 

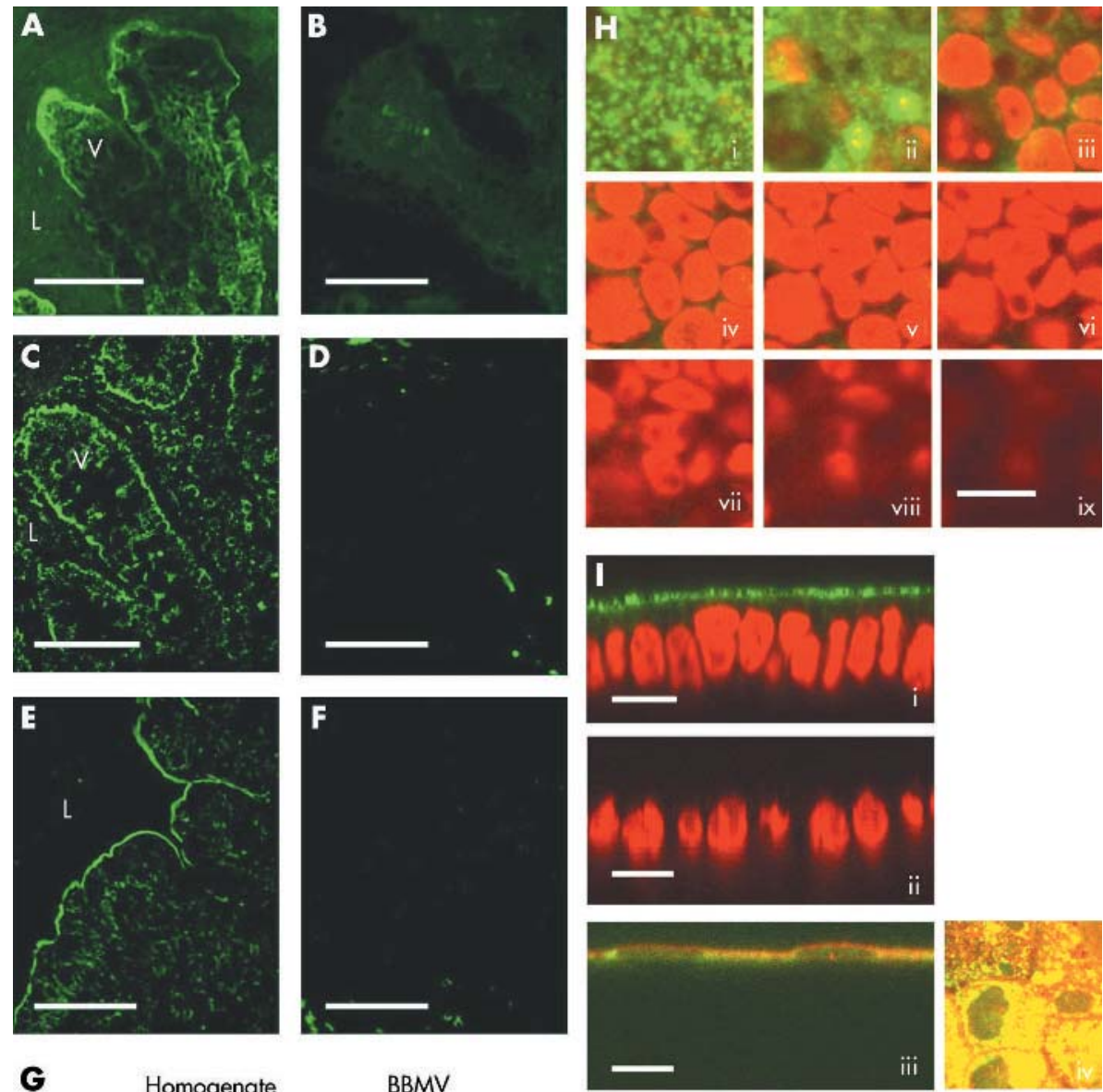

G

BBMV

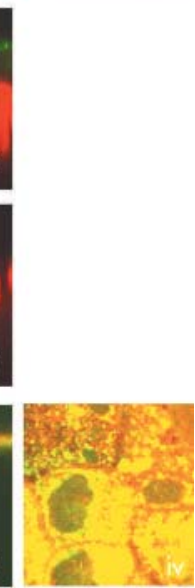

$60 \mathrm{kDa}$
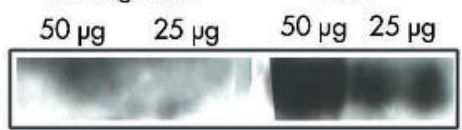

Figure 3 Immunolocalisation of SLC30A5 in human intestine and in Caco-2 cells. Binding of the primary antibody was detected using a FITC conjugated secondary antibody and is shown in green. (A) Section of human jejunum. (C) Section of human ileum. (E) Section of human colon. (B, D, F) Corresponding negative controls stained with secondary antibody only. V, villus; L, lumen; scale bar $50 \mu \mathrm{m}$. (G) Result of immunoblotting using antiSLC30A5 antibody samples of total mucosal homogenate and brush border membrane vesicles ( $50 \mu \mathrm{g}$ and $25 \mu \mathrm{g}$ of protein, as indicated) prepared from human jejunum. A predominant band of approximate $60 \mathrm{kDa}$ molecular weight was observed in the brush border membrane vesicle preparation. $(\mathrm{H}$, I) Staining in Caco-2 cells. Scale bar $25 \mu \mathrm{m}$. (H) Consecutive Z sections (parallel to the plane of the monolayer) captured by confocal laser scanning microscopy passing from the apical (i) to the basal (ix) surface of the cell monolayer. SLC30A5 staining is shown in green. Nuclei are stained with propidium iodide and are shown in red. (I) Images (i), (ii), and (iii) are XZ sections (perpendicular to the plane of the monolayer). In images (i) and (ii), nuclei are stained with propidium iodide and are shown in red. Image (iii) shows colocalisation of anti-SLC30A5 immunoreactivity and alkaline phosphatase activity (red). Image (iv) shows colocalisation of anti-SLC3OA5 immunoreactivity (green) and alkaline phosphatase activity (red) as a projected Z series viewed from above the cell monolayer.

after zinc relative to an arbitrarily assigned value of 1 for the corresponding value after placebo, based on analysis of two different loadings, as shown in fig 1), with the zinc supplement reducing expression to levels that were barely detectable by immunoblotting. For SLC30A5 the difference was approximately 1.8 -fold $(0.56(0.09)$ after zinc relative to an arbitrarily assigned value of 1 for the corresponding value after placebo, based on analysis of two different loadings, as shown in fig 1). SLC39A4 protein levels in the placebo sample were at the lower limit of detection by immunoblotting and the protein was undetectable after zinc supplementation.

Antibodies used in the study were validated by demonstrating that bands observed on immunoblots were specific and that the antibodies were reactive towards the corresponding target protein when expressed in a heterologous system. On immunoblots of protein extracted from Caco-2 cells, anti-SLC30Al, anti SLC30A5, and anti-SLC39A4 antibodies revealed protein bands that comigrated with the predominant band revealed by the same antibodies on immunoblots of human small intestinal mucosa. For antiSLC30Al and anti-SLC30A5, these immunoreactive bands were out-competed by the immunising peptide (fig 2A, C). For SLC39A4, for which immunising peptide was unavailable, the band was detected by serum from a rabbit immunised with the peptide but not with normal rabbit serum (fig 2E). When used against protein extracted from Caco- 2 cells on immunoblots, all antibodies revealed bands additional to the reproducible band comigrating with the band detected in human small intestinal mucosa using the same antibody. However, with the exception of a band of apparent molecular weight $35 \mathrm{kDa}$ detected in Caco-2 cells, but not in human intestinal mucosa, by the anti-SLC30Al antibody (fig 2A, fig 4C), the additional bands detected in Caco- 2 cells were weaker and were not reproducible between different blots and are presumed to be background. SLC39A4 and the $\mathrm{C}$ terminal region of SLC30Al, including the sequence of the immunising peptide, were expressed from 
the vector pEGFPN as $\mathrm{N}$ terminal fusions to GFP in transfected COS-7 cells. On immunoblots of protein extracted from the transfected cells, the SLC30Al and SLC39A4 antibodies revealed immunoreactive bands that were absent from COS-7 cells transfected with vector only (fig 2B, F). Reactivity of the anti-SLC30A5 antibody against expressed SLC30A5 protein was demonstrated in Xenopus laevis oocytes. Oocytes injected with SLC30A5 cRNA expressed a protein that was detected on immunoblotting with the anti-SLC30A5 antibody that was absent from oocytes injected with an equal volume of water (fig 2D).

A role for SLC30Al, SLC30A5, and SLC39A4 in zinc absorption has been indicated by functional studies ${ }^{1048}$ and a role for SLC30Al and SLC39A4 is supported by the respective basolateral and apical locations in the enterocyte of the corresponding rodent proteins, as established by immunohistochemisty. ${ }^{97}$ Existing evidence for apical localisation of SLC30A5 however is based on studies in Caco-2 cells transfected with a tagged construct corresponding to one of two splice variants of the SLC30A5 gene. ${ }^{45}$ Therefore, we examined directly in sections of human small intestine and in Caco- 2 cells the localisation of SLC30A5 using an antipeptide antibody predicted to be cross reactive with both SLC30A5 splice variants. The results are shown in fig 3 and confirm apical localisation of SLC30A5 in Caco-2 cells and in human enterocytes and colonocytes. Expression of SLC30A5 appears to be approximately equal along the length of the intestine. Enrichment of the $60 \mathrm{kDa}$ SLC30A5 specific band observed by immunoblotting in apical brush border membrane vesicles prepared from human jejunal mucosa compared with total mucosal homogenate (fig 3G) further confirms localisation of SLC30A5 in human small intestine at the apical enterocyte membrane.

Previously, we examined in Caco-2 cells the response of zinc transporter mRNAs to a change in the extracellular zinc concentration from $3 \mu \mathrm{M}$ to $100 \mu \mathrm{M}^{4}{ }^{12}$ and observed upregulation at the higher zinc concentration of SLC30Al and SLC30A5. However, the intestinal luminal zinc concentration following a meal is estimated to be in the order of $100 \mu \mathrm{M}^{4}$ and hence these experimental conditions are unlikely to reflect the response of enterocytes to an increased luminal concentration of zinc when dietary zinc intake is already adequate, as in our subjects. Therefore, we determined the effect of increasing the zinc concentration of the culture medium from $100 \mu \mathrm{M}$ to $200 \mu \mathrm{M}$ over three days on expression of SLC30Al, SLC30A5, and SLC39A4 in CaCo-2 cells at both the mRNA and protein levels. Comparison by semiquantitative RT-PCR of levels of all three mRNA species revealed a decrease at the higher zinc concentration (fig 4B). Immunoblotting of total cell protein revealed that levels of the corresponding proteins were also reduced at the higher zinc concentration, commensurate with our observations in zinc supplemented human subjects (fig 4C). We also confirmed the previously observed increase in SLC30Al and SLC30A5 mRNAs in Caco-2 cells on increasing the zinc concentration of the culture medium from $3 \mu \mathrm{M}$ to $100 \mu \mathrm{M}$ and investigated the effect of this change in culture conditions on SLC39A4 mRNA levels, which were unchanged (fig 4A). In parallel with increased levels of the corresponding mRNA, SLC30Al protein was increased in Caco-2 cells at $100 \mu \mathrm{M}$ compared with $3 \mu \mathrm{M}$ zinc, while SLC39A4 protein levels were reduced (fig 4C).

\section{DISCUSSION}

We examined the response to dietary zinc supplementation of mRNAs corresponding to a range of zinc transporters in human small intestine in vivo. Regulation of the transporters SLC30Al, SLC30A5, and SLC39A4, located at the basolateral (SLC30Al) and apical (SLC30A5 and SLC39A4) membranes of the enterocyte, was also examined at the protein level. The use of patients who had previously undergone ileostomy allowed sampling of the small intestinal mucosa via the stoma. Studies involving sampling of the intestinal mucosa
A

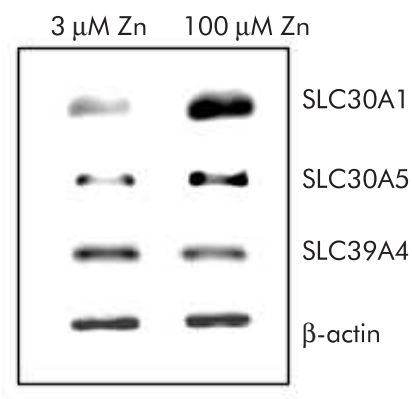

C

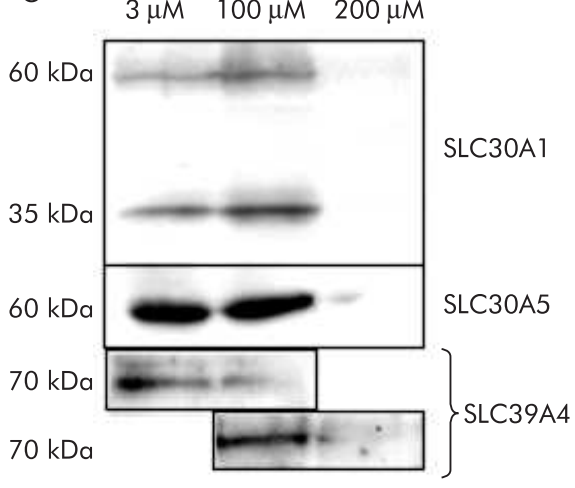

B

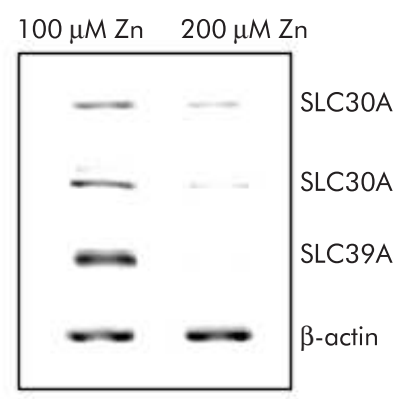

D

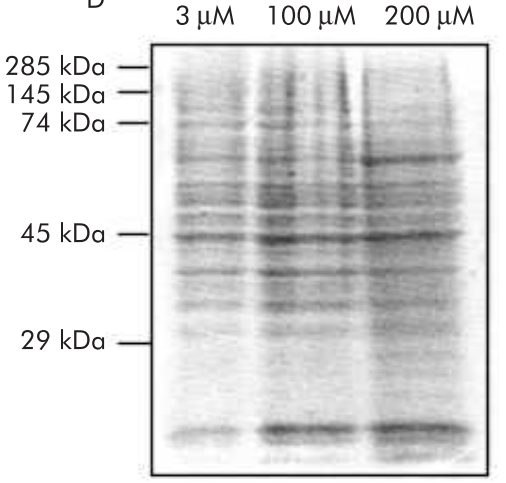

Figure 4 Regulation by zinc of SLC30A1, SLC30A5, and SLC39A4 expression in Caco-2 cells. (A, B) Reverse transcription-polymerase chain reaction (RT-PCR) products generated from total RNA prepared from Caco-2 cells using primers specific to the transcripts indicated. Products generated from RNA prepared from cells grown for three days at 3, 100, and $200 \mu \mathrm{M} \mathrm{ZnCl}_{2}$ are shown, as indicated. For each transcript, PCR was carried out over a number of cycles such that the rate of product formation did not reach a plateau, and thus band intensity is representative of the quantity of the specific transcript in the RNA sample. (C) Analysis by immunoblotting using antipeptide antibodies of expression in Caco-2 cells of SLC30A1, SLC30A5, and SLC39A4. Each sample of $7 \mu \mathrm{g}$ of protein, as determined by Bradford analysis, was resolved by sodium dodecyl sulphatepolyacrylamide gel electrophoresis (SDS-PAGE) prior to blotting. The results of analysis of samples prepared from Caco- 2 cells grown for three days at 3,100 , and $200 \mu \mathrm{M} \mathrm{ZnCl}$ are shown, as indicated. (D) A replica SDSPAGE gel, loaded as for the experiments shown in $(C)$, stained with Coomassie brilliant blue to demonstrate equal sample loading. The position of molecular weight standards is indicated. 
are required to understand the regulation of intestinal nutrient transporters by specific dietary components in human subjects, including zinc homeostatic mechanisms. Many published studies on quantification of digestion, ${ }^{25}{ }^{26}$ as well as studies involving quantification of mineral absorption (including zinc) 27-29 $^{29}$ the small intestine, have employed the ileostomy model and it is considered to be a reliable model to reflect small bowel absorption. ${ }^{30}$ All participants were free of any symptoms of previous inflammatory bowel disease. None of the drugs taken by any participant during the trial is known to alter zinc metabolism and the crossover design of our study ensured that any possible influences of medication on zinc transporter expression should not interfere with the detection of changes in the relative level of expression.

In comparison with dietary reference values for zinc, dietary zinc intakes in the human subjects studied (mean $9.2 \mathrm{mg} /$ day for men and $7.5 \mathrm{mg} /$ day for women over both trial periods and not including the supplement) exceeded the UK Estimated Average Requirement of $7.3 \mathrm{mg} /$ day for men and $5.5 \mathrm{mg} /$ day for women ${ }^{31}$ and were similar to reported mean zinc intakes in British adults of $10.2 \mathrm{mg} / \mathrm{day}$ and $7.4 \mathrm{mg} /$ day by men and women, respectively. ${ }^{32}$ These zinc intakes are considered reliable because there was no evidence of underreporting of food intake; estimated energy intakes as multiples of predicted basal metabolic rate were 1.62 (SD 0.45 ) and 1.42 (SD 0.37) for placebo and zinc supplemented periods, respectively. These energy intakes match energy needs for UK adults ${ }^{31}$ and paired $t$ test analysis showed that the means for the two treatment periods were not significantly different $(\mathrm{p}=0.06)$.

We observed that supplementation with $25 \mathrm{mg}$ zinc for 14 days resulted in downregulation of SLC30Al mRNA and of SLC30Al, SLC30A5, and SLC39A4 protein in human small intestine. These regulatory responses were mirrored in the Caco-2 cell line when the zinc concentration of the culture medium was increased from $100 \mu \mathrm{M}$ to $200 \mu \mathrm{M}$, representing an increase above the calculated luminal zinc concentration in subjects consuming a zinc adequate $\operatorname{diet}^{4}$ and hence mimicking in vitro the design of our in vivo study. In addition, parallel decreases in SLC30A5 and SLC39A4 mRNAs were observed at the higher zinc concentration in the cell line model, but not in human small intestine, possibly reflecting a higher level of exposure to zinc in cultured cells or differences in regulatory properties between the cell line model and in vivo.

In the rat, zinc supplementation resulted in upregulation of Slc30al mRNA in the small intestine. ${ }^{911}$ The level of dietary zinc supplementation in these studies, at sixfold greater than a zinc adequate diet, was comparable to the present study in human subjects where the zinc supplement resulted in an approximate 4.5 -fold increase in dietary zinc intake and was given over a similar one week ${ }^{9}$ or identical two week ${ }^{11}$ period of time. The argument that the apparent difference in zinc transporter response to zinc supplementation in rat and human systems is a result of different supplementation regimens is not therefore supported. Rather, the evidence suggests that the response to dietary zinc supplementation in rats is fundamentally different to that in humans. One week's supplementation of the diet with zinc at a sixfold increased dose above a zinc adequate diet resulted in a significant increase in serum zinc in the rat. ${ }^{9}$ This finding contrasts with our own observations in humans where zinc supplementation resulted in no significant change in plasma or serum zinc concentration. These observations may also indicate a fundamental difference between rats and humans in terms of the regulatory responses to a zinc challenge. However, this increase in serum zinc in zinc supplemented rats has not been observed consistently. ${ }^{11}$
Regulation by dietary zinc of Slc30a5 in the intestine in rodent models has not yet been reported. The effect of a zinc supplemented diet on intestinal Slc39a4 protein abundance has not been examined directly but zinc supplementation was reported to reverse a zinc induced increase in Slc39a4 mRNA $^{8}$ : hence the data available for rodent models is broadly in agreement with our observations both in human subjects and in the Caco-2 cell line that SLC39A4 is less abundant at higher zinc intakes. In addition, in pregnant mice, a zinc deficient diet induced accumulation of Slc39a4 protein at the apical surface of enterocytes, which was reversed on switching to a zinc supplemented diet, indicating an effect on intracellular trafficking. ${ }^{8}$

It is well established that increased transcription of certain genes, including SLC30Al, in response to increased zinc availability, involves the zinc regulated transcription factor MTFl. ${ }^{33}$ However, a mechanism to account for downregulation of mRNA expression in response to increased zinc availability has not yet been described in mammalian systems and might potentially involve effects on gene transcription and/or mRNA stability. In yeast, the transcription factor Zaplp is inhibited by zinc and controls transcription of the zinc transporter genes ZRT1 and ZRT2 which are therefore upregulated in response to zinc deficiency. ${ }^{35}$ While we observed in our subjects downregulation of SLC30Al in the small intestine at both the RNA and protein levels in response to zinc supplementation, we detected downregulation of SLC30A5 and SLC39A4 at only the protein level. Similarly, dissociated effects of dietary zinc on levels of rat Slc30al mRNA and protein have been reported. In rat small intestine, Slc30al mRNA levels were increased by $50 \%$ on dietary zinc supplementation whereas the corresponding protein in the small intestine was increased by only $10 \%$. Similarly, an acute oral zinc dose increased levels of rat small intestinal Slc30al mRNA eightfold without any measurable effect on corresponding protein levels. ${ }^{9}$ In contrast, an acute oral zinc dose increased Slc30al protein in rat liver without any measurable effect on Slc30al mRNA levels. ${ }^{9}$ Reduction in SLC30A5 and SLC39A4 protein levels in the absence of a parallel decrease in the corresponding mRNA species might result from reduced translation, increased protein degradation, or both. There is a paucity of knowledge about mechanisms through which zinc might interact with the components of protein translation or degradation to explain the observed reduction in SLC30A5 and SLC39A4 protein in the intestinal mucosa following zinc supplementation. Although inhibition of protein synthesis by zinc induced phosphorylation of the alpha subunit of the translation eukaryotic initiation factor-2 (eIF-2alpha) has been reported, ${ }^{36}$ such an effect would be global rather than specific. It has been demonstrated that zinc stimulated degradation of the yeast plasma membrane zinc transporter ZRTl is mediated through ubiquitination and subsequent endocytosis. ${ }^{37}$ Speculatively, such a mechanism might underlie the observed response to zinc supplementation of SLC30A5 in human intestine but there is no evidence to support this at present.

The increase in MT mRNA in the small intestine of our subjects following dietary zinc supplementation is commensurate with the response observed in the rat. ${ }^{9}$ The absence of a parallel increase in monocyte metallothionein mRNA levels may indicate either that this measure is a relatively insensitive indicator of zinc status or that the zinc status of the subjects was not altered by the intervention. This lack of responsiveness of human monocyte MT mRNA to dietary zinc supplementation contrasts with the findings of other studies. ${ }^{24}{ }^{38}$ Factors that may account for this difference include the use in other studies of a higher daily dose of $50 \mathrm{mg}$ zinc $^{24}$ or, where the daily zinc supplement was only 
$15 \mathrm{mg},{ }^{38}$ the use of only male subjects of a younger age than our own volunteers who refrained from consumption of zinc rich foods prior to the study period.

Confirmation in the present study of the localisation in human intestine of SLC30A5, a transporter established to mediate cellular zinc uptake, ${ }^{4}$ at the enterocyte/colonocyte apical membrane, along with established expression patterns of rodent Slc30al ${ }^{9}$ and Slc39a $4^{7}$ at the enterocyte basolateral and apical membranes, respectively, provides a rationale for the regulatory responses of these transporters to zinc supplementation observed in this study. The subcellular location and mode of action of all three transporters are consistent with a role in the absorption of luminal zinc. Furthermore, the association between mutations in the SLC39A4 gene and the inherited deficiency in zinc absorption, acrodermatitis enteropathica, ${ }^{67}$ establishes a functional role for SLC39A4 in dietary zinc uptake. Therefore, the observed downregulation of SLC30Al, SLC30A5, and SLC39A4 expression in response to an increased dietary zinc load would seem to be a response that contributes to maintenance of zinc homeostasis.

Copper deficiency, resulting from impaired intestinal absorption, is a well established clinical outcome of prolonged dietary supplementation with high doses of zinc. ${ }^{39} 40$ The metal ion specificity of the zinc transporters measured in the present study remains to be defined. Should copper be an additional substrate for any of the transporters studied here, reduced expression in response to zinc supplementation, in addition to potential direct competition between the two metal ions for transport, may contribute mechanistically to this outcome.

A rationale for the apparent difference in regulatory profiles between the two apical intestinal zinc transporters emerges if one considers their difference in affinity for zinc. SLC39A4 is a relatively high affinity zinc uptake transporter, with the $\mathrm{K}_{\mathrm{m}}$ for the mouse protein reported to be $1.6 \mu \mathrm{M}^{8}$ and its expression level and plasma membrane localisation appear to be upregulated under conditions of zinc deficiency to maximise uptake under conditions of low luminal zinc concentration. SLC30A5, on the other hand, is functional only at higher concentrations of zinc, ${ }^{4}$ and therefore increasing or even maintaining expression in response to a reduced dietary zinc supply would be energetically wasteful. Downregulation of both transporters under conditions of dietary zinc excess would prevent the absorption of surplus zinc.

Expression of SLC30A5 at a constant level along the cephalocaudal axis of the intestine, as demonstrated in the present study, is commensurate with dietary zinc absorption in the distal as well as the proximal intestine. Although the majority of zinc absorption from an oral dose occurs in the proximal intestine, this phenomenon is probably a function of the increased level of zinc presentation to the epithelial cells in this region. The capacity of rat colon for zinc absorption appears to be at least as high as more proximal regions. ${ }^{41-43}$

The apparent molecular weight on SDS-PAGE of proteins in human small intestinal mucosa and in Caco-2 cells recognised by the anti-SLC30Al (60 kDa), anti-SLC30A5 (60 kDa), and anti-SLC39A4 (70 kDa) antipeptide antibodies used in this study are close to the molecular weight predicted from the corresponding cDNA sequences $(58 \mathrm{kDa}$, $56 \mathrm{kDa}$, and $72 \mathrm{kDa}$, respectively), indicating that the proteins are not glycosylated in the intestine. An additional band, of approximately $35 \mathrm{kDa}$, revealed by the anti-SLC30Al antibody in Caco-2 cells, but not detected in human intestinal mucosa, may be a product of proteolytic degradation of the higher molecular weight species or of alternative RNA splicing.

To conclude, we have established responses to dietary zinc supplementation of the zinc transporters SLC30Al, SLC30A5, and SLC39A4 in human small intestine in vivo that are commensurate with a contribution to zinc homeostasis through regulation of dietary zinc absorption and which are observed also in Caco-2 cells maintained under zinc supplemented compared with zinc adequate conditions. The study is, to our knowledge, the first to examine regulation by a specific nutrient of membrane transporters in human small intestine in vivo.

\section{ACKNOWLEDGEMENTS}

This study was funded by the UK Biotechnology and Biological Sciences Research Council, grant reference 13D/1 1912. We thank Dr Sebastien Kury, LEPA, Nantes, France, for supplying anti-SLC39A4 antibody.

\section{Authors' affiliations}

R A Cragg*, S R Phillips*, J M Piper, D Ford, Institute for Cell and Molecular Biosciences, Human Nutrition Research Centre, University of Newcastle, Newcastle upon Tyne, UK

J C Mathers, School of Clinical Medical Sciences, Human Nutrition Research Centre, University of Newcastle, Newcastle upon Tyne, UK J S Varma, School of Surgical and Reproductive Sciences, University of Newcastle, Newcastle upon Tyne, UK

F C Campbell, Department of Surgery, The Queen's University of Belfast, Institute of Clinical Science, Grosvenor Road, Belfast, UK

${ }^{*} \mathrm{R}$ A Cragg and S R Phillips contributed equally to this work.

Conflict of interest: None declared.

\section{REFERENCES}

1 Hirst BH. Dietary regulation of intestinal nutrient carriers. Proc Nutr Soc 1993:52:315-24.

2 Ferraris RP, Diamond J. Regulation of intestinal sugar transport. Physiol Rev 1997:77:257-302.

3 Gunshin H, Mackenzie B, Berger UV, et al. Cloning and characterization of a mammalian proton-coupled metal-ion transporter. Nature 1997;388:482-8

4 Cragg RA, Christie GR, Phillips SR, et al. A novel zinc-regulated human zinc transporter, SLC30A5, is localised to the enterocyte apical membrane. J Biol Chem 2002;277:22789-97.

5 Kambe T, Narita H, Yamaguchi-lwai Y, et al. Cloning and characterization of a novel mammalian zinc transporter, zinc transporter 5, abundantly expressed in pancreatic beta cells. J Biol Chem 2002;277:19049-55.

6 Kury S, Dreno B, Bezieau S, et al. Identification of SLC39A4, a gene involved in acrodermatitis enteropathica. Nat Genet 2002;31:239-40.

7 Wang K, Zhou B, Kuo YM, et al. A novel member of a zinc transporter family is defective in acrodermatitis enteropathica. Am J Hum Genet 2002;71:66-73.

8 Dufner-Beattie J, Wang F, Kuo YM, et al. The acrodermatitis enteropathica gene ZIP4 encodes a tissue-specific, zinc-regulated zinc transporter in mice. J Biol Chem 2003;278:33474-81.

9 McMahon RJ, Cousins RJ. Regulation of the zinc transporter ZnT-1 by dietary zinc. Proc Natl Acad Sci U S A 1998;95:4841-6.

10 Palmiter RD, Findley SD. Cloning and functional charaterisation of a mammalian zinc transporter that confers resistance to zinc. $E M B O J$ 1995; 14:639-49.

11 Liuzzi JP, Blanchard RK, Cousins RJ. Differential regulation of zinc transporter 1,2 and 4 mRNA expression by dietary zinc in rats. J Nutr 2001;131:46-52.

12 Phillips SR, Russi RM, Cragg RA, et al. The effect of zinc on expression in human intestinal and placental cell lines of zinc transporter mRNAs. Proc Nutr Soc 2002;61:46A.

13 Palmiter RD, Cole TB, Findley SB. ZnT-2, a mammalian protein that confers resistance to zinc by facilitating vesicular sequestration. EMBO J 1996; 15:1784-91.

14 Murgia C, Vespignani I, Cerase J, et al. Cloning, expression and vesicular localization of zinc transporter Dri 27/Znt4 in intestinal tissue and cells. Am J Physiol 1999;277:G1231-9.

15 Dufner-Beattie J, Langmade SJ, Wang F, et al. Structure, function and regulation of a subfamily of mouse zinc transporter genes. $J$ Biol Chem 2003;278:50142-50.

16 Menard MP, Cousins RJ. Zinc transport by brush border membrane vesicles from rat intestine. J Nutr 1983;113:1434-42.

17 Smith KT, Cousins RJ. Quantitative aspects of zinc absorption by isolated, vascularly perfused rat intestine. J Nutr 1980;110:316-23.

18 Hoadley JE, Leinart AS, Cousins RJ. Kinetic analysis of zinc uptake and serosal transfer by vascularly prefused rat intestine. Am J Physiol 1987;252:G825-31.

19 Nelson M, Atkinson M, Meyer J. Photographic atlas of food portion sizes. London: MAFF Publications, 1997. 
20 Food Standards Agency. McCance and Widdowson's the composition of foods, sixth summary edn. Cambridge: Royal Society of Chemistry, 2002.

21 Shirazi-Beechey SP, Davies AG, Tebbutt K, et al. Preparation and properties of brush-border membrane vesicles from human small intestine. Gastroenterology 1990;98:676-85.

22 Dyer J, Wood IS, Palejwala A, et al. Expression of monosaccharide transporters in intestine of diabetic humans. Am J Physiol 2002;282:G241-8.

23 Shirazi SP, Beechey RB, Butterworth PJ. The use of potent inhibitors of alkaline phosphatase to investigate the role the enzyme in intestinal transport of inorganic phosphate. Biochem J 1980;194:803-9.

24 Sullivan VK, Cousin RB. Competitive reverse transcriptase-polymerase chain reaction shows that dietary zinc supplementation in humans increases monocyte metallothionein mRNA levels. J Nutr 1997;127:694-8.

25 Netherwood T, Martin-Orue SM, O'Donnell AG, et al. Assessing the survival of transgenic plant DNA in the human gastrointestinal tract. Nat Biotech 2004;22:204-9.

26 Andersson $\mathrm{H}$. The ileostomy model for the study of carbohydrate digestion and carbohydrate effects on sterol excretion in man. Eur J Clin Nutr 1992;46:S69-76.

27 Ellegard L, Andersson H, Bosaeus I. Inulin and oligofructose do not influence the absorption of cholesterol, or the excretion of cholesterol, $\mathrm{Ca}, \mathrm{Mg}, \mathrm{Zn}, \mathrm{Fe}$, or bile acids but increases energy excretion in ileostomy subjects. Eur J Clin Nutr 1997;51:1-5.

28 Sandberg AS, Andersson H, Bosaeus I, et al. Alginate, small bowel sterol excretion, and absorption of nutrients in ileostomy subjects. Am J Clin Nutr 1994;60:751-6.

29 Sandstrom B, Andersson H, Kivisto B, et al. Apparent small intestinal absorption of nitrogen and minerals from soy and meat-protein-based diets. A study on human ileostomy subjects. J Nutr 1986;116:2209-18.

30 Andersson HB, Ellegard LH, Bosaeus IG. Nondigestibility characteristics of inulin and oligofructose in humans. J Nutr 1999;129:1428-30S.

31 Department of Health. Report in health and social subjects 41. Dietary reference values for food energy and nutrients for the United Kingdom. Committee on Medical Aspects of Food Policy. London: HMSO, 1991.
32 Office for National Statistics. The National Diet and Nutrition Survey: adults ages 19 to 64 years (volume 3; vitamin and mineral intake and urinary analytes). London: HMSO, 2003

33 Heuchel R, Radtke F, Georgiev O, et al. The transcription factor MTF-1 is essential for basal and heavy metal-induced metallothionein gene expression. EMBO J 1994;13:2870-5.

34 Langmade SJ, Ravindra R, Daniels PJ, et al. The transcription factor MTF-1 mediates metal regulation of the mouse SLC30A1 gene. J Biol Chem 2000;275:34803-9.

35 Zhao H, Eide DJ. Zaplp, a metalloregulatory protein involved in zincresponsive transcriptional regulation in Saccharomyces cerevisiae. Mol Cell Biol 1997; 17:5044-52

36 Alirezaei M, Nairn AC, Glowinski J, et al. Zinc inhibits protein synthesis in neurons. Potential role of phosphorylation of translation initiation factor2alpha. J Biol Chem 1999;274:32433-8.

37 Gitan RS, Eide DJ. Zinc-regulated ubiquitin conjugation signals endocytosis of the yeast ZRT1 zinc transporter. Biochem J 2000;346:329-36.

38 Cao J, Cousins RJ. Metallothionein mRNA in monocytes and peripheral blood mononuclear cells and in cells from dried blood spots increases after zinc supplementation of men. J Nutr 2000;130:2180-7.

39 Gyorffy EJ, Chan H. Copper deficiency and microcytic anaemia resulting from prolonged ingestion of over-the-counter zinc. Am J Gastroenterol 192, 87:1054-5.

40 Fiske DN, McCoy HE, Kitchens CS. Zinc-induced sideroblastic anemia: report of a case, review of literature, and description of the hematologic syndrome. Am J Hematol 1994;46:147-50.

41 Seal CJ, Mathers JC. Intestinal zinc transfer by everted gut sacs from rats given diets containing different amounts and types of dietary fibre. $\mathrm{Br} J$ Nutr 1989:62:151-63.

42 Gisbert-Gonzalez SL, Torres-Molina F. Zinc uptake in five sectors of the rat gastrointestinal tract: kinetic study in the whole colon. Pharm Res 1996;13:1154-61.

43 Condomina J, Zornoza-Sabina T, Granero L, et al. Kinetics of zinc transport in vitro in rat small intestine and colon: interaction with copper. Eur J Pharm Sci 2002; 16:289-95.

\section{EDITOR'S QUIZ: GI SNAPSHOT}

\section{An unusual abdominal mass}

\section{Clinical presentation}

A 70 year old woman presented with a painless abdominal mass. She had a background of previous oesophageal carcinoma with liver metastases with a remarkably long disease free interval of over three years after complete response to local radiotherapy and systemic chemotherapy. Cholecystectomy had been performed for cholelithiasis more than 10 years previously.

Computed tomographic (CT) scan demonstrated a large cystic structure arising from the bowel mesentery (fig l). A presumed diagnosis of recurrent disease was made. In the absence of other evidence of metastatic disease on CT of the chest, abdomen, and pelvis, surgical resection was planned.

\section{Question}

What did laparotomy reveal as the cause of the abdominal mass?

See page 514 for answer

This case is submitted by:

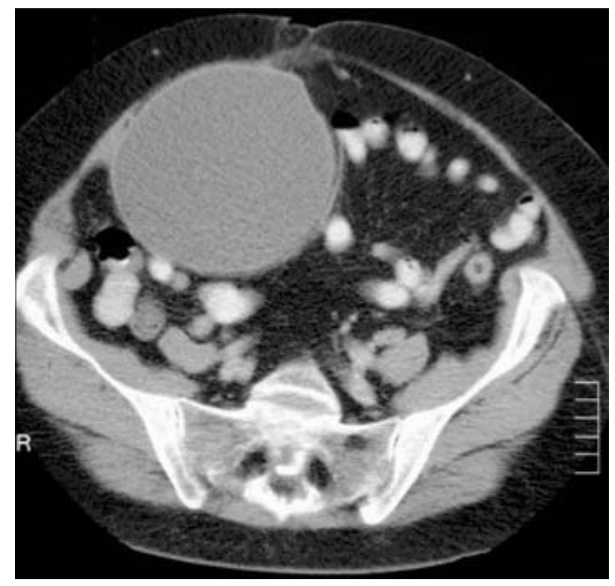

Figure 1 Computed tomographic scan of the abdomen.

P Grimison, D Goldstein Department of Medical Oncology, Prince of Wales Hospital, Randwick NSW, Australia

B Yeo

Department of Surgery, Prince of Wales Hospital, Randwick NSW, Australia

Correspondence to: Dr P Grimison, Department of Medical Oncology, Prince of Wales Hospital, Barker St, Randwick NSW 2031, Australia; grimisonp@sesahs.nsw.gov.au

doi: 10.1136/gut.2004.051342 